\title{
A model of an isolated magnetic flux tube in a stratified atmosphere
}

\author{
By WEN-RUI HU \\ Institute of Mechanics, Chinese Academy of Science, Beijing, China
}

(Received 20 May 1986 and in revised form 2 July 1986)

A two-dimensional model of a magnetic flux tube confined in a gravitational stratified atmosphere is discussed. The magnetic field in the flux tube is assumed to be force-free. By using the approximation of large scale height, the problem of a free boundary with nonlinear conditions may be reduced to one involving a fixed boundary. The two-dimensional features are obtained by applying the perturbation method and adopting the Lüst-Schlüter model as the basic state. The results show that the configuration of a flux tube confined in a gravitational stratified atmosphere is divergent, and the more twisted the magnetic field, the more divergent is the flux tube.

\section{Introduction}

An isolated magnetic flux tube confined in a stratified atmosphere is the typical configuration in astrophysical environments. Observations of the solar atmosphere show that very little of the net magnetic flux can escape, being concentrated into isolated tubes (see, for example, the reviews of Parker (1979, ch. 8) and Spruit (1981)). On the other hand, the isolated flux tube is confined in the atmosphere, where thermodynamic quantities can be described by the stratified model. This may be the situation for many magnetic configurations in the stellar atmosphere. In order to understand the features of an isolated flux tube, we construct a model and analyse the influence of the atmospheric scale height on the equilibrium of the magnetic field.

A typical isolated axisymmetric flux tube in the cylindrical co-cordinate system $(r, \theta, z)$ is drawn in figure 1. The magnetic flux is concentrated in the region confined by the boundary $\Gamma$. It is believed that the radius of the boundary increases with the height $z$, as the atmospheric pressure decreases. If a uniform pressure model of the atmosphere is adopted, the flux tube reduces to a cylinder, and we could use a one-dimensional model where the magnetic configuration depends only on the co-ordinate $r$, as in the Lüst-Schlüter model (1954). However, the two-dimensional configuration is more interesting. Similarity solutions were suggested by Deinzer (1965), Yun (1970) and Osherovich (1984). In addition to the limitation due to the similarity assumption, these models deal with continuous fields without a definite boundary $\Gamma$. Wilson (1977) employed a two-dimensional magnetostatic model of a twisted flux tube, and $\mathrm{Hu}$ (1981) used the perturbation method to obtain a formal solution for a slender flux tube. Recently, Deinzer et al. (1984) calculated numerically the interaction of the flow field with an isolated flux tube.

In the present paper, we discuss the case of large atmospheric scale height, 


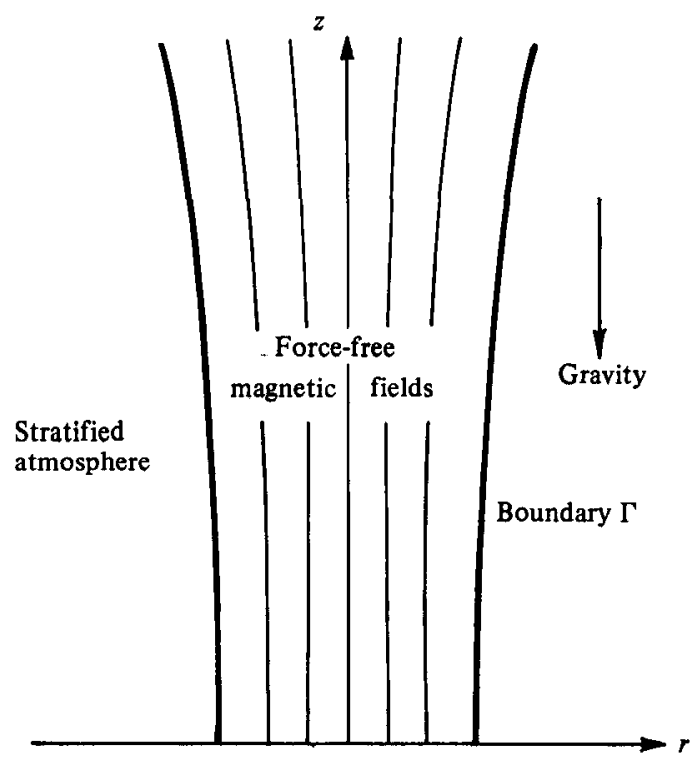

FIoURE 1. Model of an isolated magnetic flux tube of force-free field confined by the stratified atmosphere.

where the deviation from uniform atmospheric pressure model is small. In this case, a perturbation method can be applied, the uniform atmospheric pressure model being taken as the zeroth-order approximation, and the influence of the atmospheric scale height being taken as first order. In the next section, we discuss the physical model and the mathematical description. The solutions given by the perturbation expansion are presented in $\S 3$. By using the nonlinear Lüst--Schlüter model for the zeroth-order situation, the first-order solution is obtained in $\$ 4$. $\$ 5$ contains a discussion and conclusions. The results show that a magnetic flux tube confined by the stratified atmosphere is an expanding tube.

\section{The physical model}

In a physical description of an isolated flux tube involves consideration of the fields in the flux tube region, the fields in the atmospheric region outside the flux tube, and the boundary condition that connects two regions. The magnetic field in the flux tube may be considered to be force-free if it is relatively strong. Our model for the atmosphere outside the flux tube is that it is stratified with constant scale height. Therefore, in the present paper, we analyse a force-free flux tube confined in the stratified atmosphere as suggested by Spruit (1981). Furthermore, as a first step, we assume that the scale height is large relative to the typical radius of the flux tube. It can be seen that the flux tube is a cylinder if the atmospheric pressure outside it is constant. The approximation of large scale height implies that the shape of the flux tube deviates only slightly from cylindrical. In this case, the perturbation method can be applied.

The force-free field may be described by

$$
\begin{aligned}
\nabla \times \mathbf{B} & =\alpha \mathbf{B}, \\
\nabla . \mathbf{B} & =0,
\end{aligned}
$$


where $\alpha$ is a function of spatial co-ordinates for the nonlinear static model. To satisfy (2.2), we introduce a magnetic streaming function $\psi$ for the axisymmetric problem,

Then (2.1) gives

$$
B=\left(\frac{1}{r} \frac{\partial \psi}{\partial z}, B_{\theta},-\frac{1}{r} \frac{\partial \psi}{\partial r}\right)
$$

where

$$
\begin{gathered}
\frac{\partial^{2} \psi}{\partial r^{2}}-\frac{1}{r} \frac{\partial \psi}{\partial r}+\frac{\partial^{2} \psi}{\partial z^{2}}+G(\psi) \frac{d G(\psi)}{d \psi}=0, \\
r B_{\theta}=G(\psi),
\end{gathered}
$$

Equation (2.4) can be solved for $\psi$ if the magnetic streaming function $G(\psi)$ and suitable boundary conditions are given.

In the region outside the flux tube, the atmosphere is in static equilibrium

$$
d p_{e} / d z=-\rho_{e} g,
$$

where we write $g=-g e_{z}$. Equation (2.7) gives the pressure distribution

$$
p_{e}(z)=p_{0} \exp \left(-\frac{z-z_{0}}{h}\right)
$$

for the isothermal ( $T_{e}=$ constant) model, with the boundary condition $p_{0}=p_{e}\left(z_{0}\right)$ and scale height $h=\mathscr{R} T_{e} / g$. These relations mean that the magnetic field inside the flux tube has no influence on the outside atmosphere.

The boundary $\Gamma$ is a 'contact' discontinuous surface. The boundary condition there is

$$
\frac{B^{2}}{8 \pi}=p_{e} \quad(\mathrm{r} \in \Gamma)
$$

By using (2.3), (2.5) and (2.8), this may be rewritten

$$
\frac{1}{8 \pi r^{2}}\left[\left(\frac{\partial \psi}{\partial z}\right)^{2}+\left(\frac{\partial \psi}{\partial r}\right)^{2}+G^{2}(\psi)\right]=p_{0} \exp \left(-\frac{z-z_{0}}{h}\right) \quad(\mathrm{r} \in \Gamma)
$$

Thus the boundary condition is nonlinear and the boundary $\Gamma$ is undetermined. So, we have a nonlinear problem with a free boundary surface, which is the main source of difficulty in considering an isolated flux tube.

To complete the mathematical formulation, we give the boundary conditions of the flux tube at $z=z_{0}$ and $z=z_{1}$,

$$
\begin{aligned}
& \psi\left(r, z_{0}\right)=\psi_{b 0}(r), \\
& \psi\left(r, z_{1}\right)=\psi_{b 1}(r),
\end{aligned}
$$

where $z_{1}>z_{0}$. Physical considerations require the boundary to be a magnetic streaming surface, so

$$
\left.\psi(r, z)\right|_{\Gamma}=\psi_{\Gamma}=\text { constant }
$$

Then, the mathematical problem is to solve (2.4) under the boundary conditions $(2.10)-(2.13)$. It is easy to see that an analytical solution can only be obtained for some simplified cases. 


\section{The approximation of large scale height}

In the uniform pressure atmosphere, the isolated flux tube may be a circular cylinder, which is described by a one-dimensional model in a cylindrical co-ordinate system. Then, all quantities depend only on the co-ordinate $r$ and the boundary $\Gamma$ is determined as a cylinder $r=r_{0}$. In the case of large atmospheric scale height, the shape of the isolated flux tube deviates only slightly from the circular cylinder. The latter is therefore considered as the basic state, and we proceed with linearized perturbation theory, writing.

or

$$
\begin{aligned}
& \mathbf{B}=\mathbf{B}_{0}+\mathbf{B}_{1} \\
& \psi=\psi_{0}+\psi_{1},
\end{aligned}
$$

where the subscriptions 0 and 1 denote, respectively, the basic state and the perturbed state, and

in the near field, where

$$
p_{e}=p_{0}+p_{1}
$$

$$
p_{1}=p_{0}\left[\exp \left(-\frac{z-z_{0}}{h}\right)-1\right]
$$

or

in the far field, where

$$
p_{e}=p_{1}
$$

$$
p_{1}=p_{0} \exp \left(-\frac{z-z_{0}}{h}\right) \text {. }
$$

In the present paper, we confine our analyses mainly to the near field region.

The nonlinear problem of the basic state is written generally as

$$
\begin{gathered}
\frac{d^{2} \psi_{0}}{d r^{2}}-\frac{1}{r} \frac{d \psi_{0}}{d r}+G_{0}\left(\psi_{0}\right) \frac{d G_{0}\left(\psi_{0}\right)}{d \psi_{0}}=0, \\
\frac{1}{r_{0}^{2}}\left[\frac{d \psi_{0}\left(r_{0}\right)}{d r}\right]^{2}+\frac{1}{r_{0}^{2}} G_{0}^{2}\left[\psi_{0}\left(r_{0}\right)\right]=8 \pi p_{e},
\end{gathered}
$$

where the boundary $\Gamma_{0}$ is $r=r_{0}$. It is easy to see that (2.13) is satisfied for the one-dimensional model. Equation (3.6) together with the boundary condition (3.7) may be solved for $\psi_{0}(r)$ if a suitable function $G_{0}\left(\psi_{0}\right)$ is given. The Lüst-Schlüter model will be applied in detail in the next section.

Now, we discuss the perturbed state. Substituting (3.1) into (2.1) and (2.2), we have

$$
\begin{gathered}
\left(\nabla \times \mathbf{B}_{0}\right) \times \mathbf{B}_{1}+\left(\nabla \times \mathbf{B}_{1}\right) \times \mathbf{B}_{\mathbf{0}}=0, \\
\nabla \cdot \mathbf{B}_{1}=0 .
\end{gathered}
$$

By using the basic state relationship

(3.8) and (3.9) reduce to

$$
\nabla \times \mathbf{B}_{0}=\alpha_{0} B_{0},
$$

$$
\begin{gathered}
\left(\frac{\partial B_{1 \theta}}{\partial z}+\frac{\alpha_{0}}{r} \frac{\partial \psi_{1}}{\partial z}\right) B_{0 z}=0 \\
\left(\frac{\partial^{2} \psi_{1}}{\partial r^{2}}-\frac{1}{r} \frac{\partial \psi_{1}}{\partial r}+\frac{\partial^{2} \psi_{1}}{\partial z^{2}}-\alpha_{0} r B_{1 \theta}\right) B_{0 z}-\left(\frac{\partial r B_{1 \theta}}{\partial r}+\alpha_{0} \frac{\partial \psi_{1}}{\partial r}\right) B_{0 \theta}=0 \\
\left(\frac{\partial B_{1 \theta}}{\partial z}+\frac{\alpha_{0}}{r} \frac{\partial \psi_{1}}{\partial z}\right) B_{0 \theta}=0
\end{gathered}
$$


Because the magnetic field is not zero in the basic state, we obtain the following relationship from (3.10) and (3.12):

$$
B_{1 \theta}+\frac{\alpha_{0}}{r} \psi_{1}=\chi(r)
$$

where $\chi(r)$ is an arbitrary function, which will be determined by the boundary condition at $z=z_{0}$ or $z=z_{1}$. We have

$$
\chi(r)=0
$$

there because

$$
\psi_{1}(r, 0)=0, \quad B_{1 \theta}(r, 0)=0 .
$$

In this case

$$
B_{1 \theta}=-\frac{\alpha_{0}}{r} \psi_{1} \text {. }
$$

Substituting (3.16) into (3.10), we have

$$
\frac{\partial^{2} \psi_{1}}{\partial r^{2}}-\frac{1}{r} \frac{\partial \psi_{1}}{\partial r}+\frac{\partial^{2} \psi_{1}}{\partial z^{2}}+K(r) \psi_{1}=0
$$

where the coefficient $K(r)$ is

$$
K(r)=\alpha_{0}^{2}+\frac{B_{0 \theta}}{B_{0 z}} \frac{d \alpha_{0}}{d r} .
$$

We denote the boundary $\Gamma$ by

$$
r=r_{0}+s(z)
$$

where the small term $s(z)$ is a function to be determined. The boundary condition $(2.9)$ is then

$$
\left[\frac{B_{0}^{2}(r)}{8 \pi}+\frac{B_{1}^{2}(r, z)}{8 \pi}+\frac{B_{0}(r) B_{1}(r, z)}{4 \pi}\right]_{r-r_{0}+8(z)}=p_{0}+p_{1} .
$$

By using the basic boundary condition (3.7) and the expansion for the small term $s(z)$, the perturbed boundary condition may be written as

$$
B_{0}\left(r_{0}\right) \frac{d B_{0}\left(r_{0}\right)}{d r} s(z)+\mathbf{B}_{0}\left(r_{0}\right) \cdot \mathbf{B}_{1}\left(r_{0}, z\right)=4 \pi p_{1},
$$

where we omit the terms higher than first order. Similarly, (2.13) reduces to

$$
\psi_{1}\left(r_{0}, z\right)+\frac{d \psi_{0}\left(r_{0}\right)}{d r} s(z)=0
$$

Eliminating $s(z)$ from (3.21) and (3.22), we get the condition

$$
\mathbf{B}_{0}\left(r_{0}\right) \cdot \mathbf{B}_{1}\left(r_{0}, z\right)-\frac{B_{0}\left(r_{0}\right)}{d \psi_{0}(r) / d r} \frac{d B_{0}\left(r_{0}\right)}{d r} \psi_{1}\left(r_{0}, z\right)=4 \pi p_{1},
$$

where the perturbed magnetic field may be written as

$$
\mathbf{B}_{1}(r, z)=\left(\frac{1}{r} \frac{\partial \psi_{1}}{\partial z},-\frac{\alpha_{0}}{r} \psi_{1},-\frac{1}{r} \frac{\partial \psi_{1}}{\partial r}\right) .
$$

Equation (3.17) and boundary condition (3.23) determine the magnetic stream function $\psi_{1}$, and the boundary function $s(z)$ is given by (3.22). Therefore, the problem of a free surface boundary may be solved in the approximation of large scale height. 


\section{The Lüst-Schlüter model}

The well-known Lüst-Schlüter model gives the magnetic field as

$$
B=(8 \pi)^{\frac{1}{2}}\left(0,\left(-\frac{r}{2} F^{\prime}\right)^{\frac{1}{2}},\left(F+\frac{r}{2} F^{\prime}\right)^{\frac{1}{2}}\right),
$$

where $F^{\prime}$ is the derivative of the magnetic pressure,

$$
F(r)=\frac{1}{8 \pi}\left(B_{\theta}^{2}+B_{r}^{2}\right)
$$

The boundary condition (3.7) requires

$$
F\left(r_{0}\right)=p_{0} .
$$

From the definition of the magnetic field (4.1), we have

$$
\alpha_{0}(r)=-\frac{1}{2} \frac{3 F^{\prime}+r F^{\prime \prime}}{\left[-r F^{\prime}\left(2 F+r F^{\prime}\right)\right]^{\frac{1}{2}}} .
$$

In the Lüst-Schlüter model, the function $F(r)$ must satisfy the conditions

but is otherwise arbitrary.

$$
F^{\prime}<0, \quad F+\frac{1}{2} r F^{\prime}>0,
$$

By using the Lüst-Schlüter model, we obtain the equation and boundary conditions for the perturbed magnetic stream function as follows:

$$
\begin{gathered}
\frac{\partial^{2} \psi_{1}}{\partial r^{2}}-\frac{1}{r} \frac{\partial \psi_{1}}{\partial r}+\frac{\partial^{2} \psi_{1}}{\partial z^{2}}+K(r) \psi_{1}=0 \\
4 \pi r_{0} F^{\prime}\left(r_{0}\right) s(z)-\left\{-4 \pi r_{0} F^{\prime}\left(r_{0}\right)\right\}^{\frac{1}{2}} \alpha_{0}\left(r_{0}\right) \psi_{1}\left(r_{0}, z\right) \\
-\left\{8 \pi\left[F\left(r_{0}\right)+\frac{1}{2} r_{0} F^{\prime}\left(r_{0}\right)\right]\right\}^{\frac{1}{2}} \frac{\partial \psi_{1}\left(r_{0}, z\right)}{\partial r}=4 \pi r_{0} p_{1} \\
s(z)=\frac{\psi_{1}\left(r_{0}, z\right)}{r_{0}\left\{8 \pi\left[F\left(r_{0}\right)+\frac{1}{2} r_{0} F^{\prime}\left(r_{0}\right)\right]\right\}^{\frac{1}{2}}}
\end{gathered}
$$

where

$$
K(r)=-\frac{2 r^{2} F^{\prime \prime \prime}\left(2 F+r F^{\prime}\right)+r F^{\prime \prime}\left(20 F+4 r F^{\prime}-r^{2} F^{\prime \prime}\right)+3 F^{\prime}\left(4 F-r F^{\prime}\right)}{4 r\left(2 F+r F^{\prime}\right)^{2}} .
$$

In accordance with the suggestion of Parker (1974), we assume

$$
F(r)=p_{0} \frac{a^{2}+r_{0}^{2}}{a^{2}+r^{2}}
$$

where $a$ is a parameter. Then, the coefficient $K(r)$ in (4.5) reduces to

$$
K(\xi)=\frac{4\left(A^{2}-\xi^{2}\right)}{\left(A^{2}+\xi^{2}\right)^{2}} \frac{1}{r_{0}^{2}}
$$

where the non-dimensional quantities are defined as

$$
\xi=\frac{r}{r_{0}}, \quad A=\frac{a}{r_{0}} .
$$


The boundary conditions (4.6) and (4.7) reduce to

$$
\frac{3 A^{2}+1}{A^{2}+1} \psi_{1}\left(r_{0}, z\right)+r_{0} A^{2} \frac{\partial \psi_{1}\left(r_{0}, z\right)}{\partial r}=-\frac{A\left(A^{2}+1\right)^{\frac{1}{2}}}{2}\left(8 \pi p_{0}\right)^{\frac{1}{2}}\left(e^{-z / h}-1\right)
$$

and

$$
s(z)=\left(A^{2}+1\right)^{\frac{1}{2}} \frac{r_{0}}{A} \frac{\psi_{1}\left(r_{0}, z\right)}{8 \pi p_{0}} .
$$

To deal with (4.5) and boundary condition (4.12), we will search for the solutions of the form

$$
\psi_{1}(r, z)=\sum_{n=0}^{\infty} R_{n}(r) Z_{n}(z)
$$

where the function $Z_{n}(z)$ is

$$
Z_{n}(z)=\sin \left(\lambda_{n} \frac{z}{h}\right)
$$

and $\lambda_{n}$ is the characteristic values. The function $\exp (-z / h)-1$ in the right-hand side of (4.12) may be written as

$$
\exp \left(-\frac{z}{h}\right)-1=\sum_{n=0}^{\infty} q_{n} \sin \left(\lambda_{n} \frac{z}{h}\right) \quad\left(z_{0} \leqslant z \leqslant z_{1}\right)
$$

where $q_{n}$ is the coefficients of the series. By using relationships (4.14)-(4.16), the following equation and boundary conditions for function $R_{n}(r)$ are obtained from (4.5), boundary condition (4.12) and the regular condition at the symmetric axis:

$$
\begin{aligned}
R_{n}^{\prime \prime}-\frac{1}{r} R_{n}^{\prime}+\left[K(r)-\frac{\lambda_{n}^{2}}{h^{2}}\right] R_{n}=0, \\
\frac{3 A^{2}+1}{A^{2}+1} R_{n}\left(r_{0}\right)+r_{0} A^{2} R_{n}^{\prime}\left(r_{0}\right)=-\frac{A\left(A^{2}+1\right)^{\frac{1}{2}}}{2}\left(8 \pi p_{0}\right)^{\frac{1}{2}} q_{n}, \\
R_{n}(0)=0, \\
R_{n}^{\prime}(0)=0,
\end{aligned}
$$

where the function $K(r)$ is given by (4.10). Equation (4.17), with boundary conditions (4.18)-(4.20), may be solved numerically, if the characteristic values $\lambda_{n}$ are determined by the boundary condition at $z=z_{1}$.

In the case of the large scale height approximation, there is a small parameter $\epsilon=r_{0} / h \ll 1$. In the near field, the left-hand side of (4.16) may be expanded as

$$
\exp \left(-\frac{z}{h}\right)-1=-\frac{z}{h}[1+O(\epsilon)]
$$

or in another form, in agreement with the right-hand side of (4.16), it can be rewritten as

$$
\exp \left(-\frac{z}{h}\right)-1=-\sin \left(\frac{z}{h}\right)[1+O(\epsilon)]
$$

Expressions (4.21) and (4.22) are valid only in the region $z / h \ll 1$. In a more extensive region, we write

$$
\exp \left(-\frac{z}{h}\right)-1=\sin \left(\lambda \frac{z}{h}\right)[1+O(\epsilon)]
$$




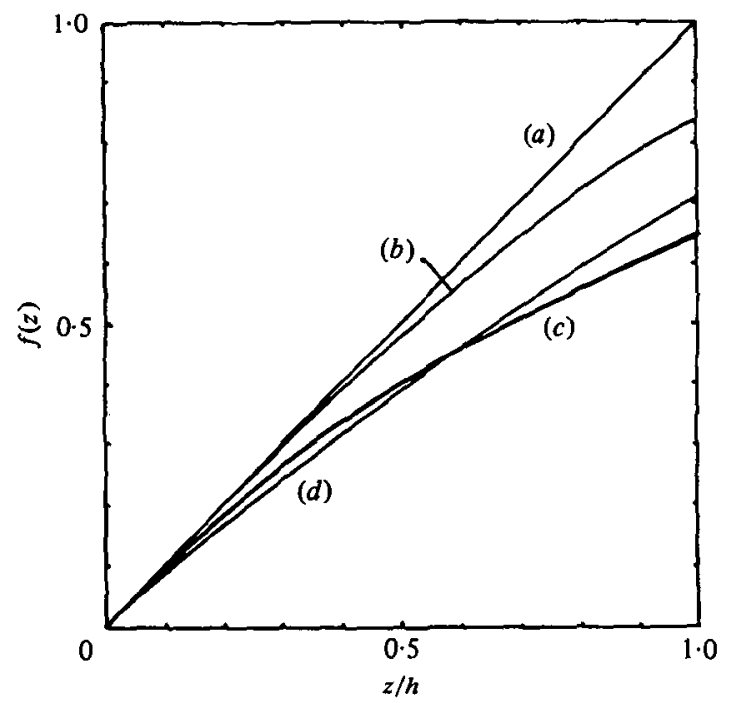

Frgure 2. Comparison of asymptotic functions. $(a) z / h,(b) \sin (z / h),(c) 1-e^{-z / h}$, (d) $\sin (0.8 z / h)$.

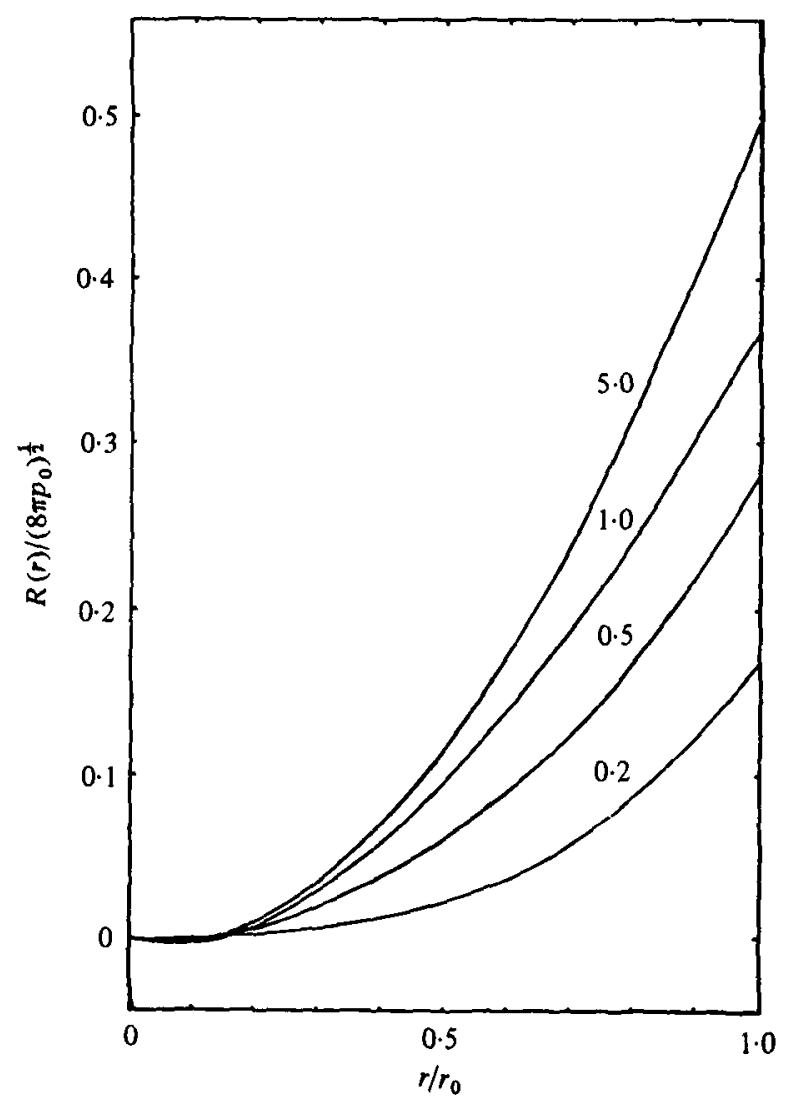

Fiqure 3. The function $R(r)$ for different values of $A$, shown against the curves. 
where $\lambda=0.8$. 'These functions are shown in figure 2 for comparison. It shows that expression (4.23) is the best choice. According to (4.23), solution (4.14) becomes

$$
\psi_{1}(r, z)=R(r) \sin \left(\lambda \frac{z}{h}\right)
$$

and it corresponds to the following conditions in comparison with expression (4.16):

$$
\left.\begin{array}{lll}
q_{0}=1, & q_{n}=0 & (n \geqslant 1), \\
\lambda_{0}=0 \cdot 8, & \lambda_{n}=0 & (n \geqslant 1) .
\end{array}\right\}
$$

Equation (4.17) is linear and homogeneous, a solution multiplied by a constant factor is still a solution of the equation. Therefore, we solve numerically the equation under the initial conditions at $r=0$, that is, conditions (4.19), (4.20) and a non-zero $R^{\prime \prime}(0)$, and then, the multiplier factor could be determined from condition (4.18). The solutions for typical values of $A$ are given in figure 3 , where we take $r_{0}=1$ and $r_{0} / h=0 \cdot 1$. The results show that $R(r)$ increases monotonically with $r$, and the larger the value of $A$, the greater is $R(r)$. By using these solutions, the magnetic fields are given as follows:

$$
\begin{gathered}
B_{r}=\frac{\lambda}{r_{0} h} \frac{R(\xi)}{\xi} \cos \left(\lambda \frac{z}{h}\right) \\
B_{\theta}=\left[8 \pi p_{0}\left(A^{2}+1\right)\right]^{\frac{1}{2}} \frac{\xi}{A^{2}+\xi^{2}}-\frac{1}{r_{0}^{2}} \frac{2 A}{A^{2}+\xi^{2}} \frac{R(\xi)}{\xi} \sin \left(\lambda \frac{z}{h}\right) \\
B_{z}=\left[8 \pi p_{0}\left(A^{2}+1\right)\right]^{\frac{1}{2}} \frac{A}{A^{2}+\xi^{2}}-\frac{1}{r_{0}^{2}} \frac{R^{\prime}(\xi)}{\xi} \sin \left(\lambda \frac{z}{h}\right)
\end{gathered}
$$

where the solutions $R(\xi)$ are shown in figure 3. The distributions of the magnetic field (4.26)-(4.28) give the two-dimensional features of the confined magnetic flux tube, and the solutions are regular in the confined region including the axis of symmetry.

\section{Discussion}

It is generally believed that a flux tube confined in a gravitationally stratified atmosphere has an expansive configuration, because the atmospheric pressure outside the tube decreases with increasing height. Mathematically, the conservation of total pressure is required to hold only at the boundary of the flux tube, where the magnetic field may be relatively either stronger or weaker, and it seems that the cross-section of the flux tube may be either divergent or convergent. However, the results of the present paper show that the flux tube is divergent for all values of $A$.

According to the boundary function (4.7) or (4.13), the flux tube is divergent if $R\left(r_{0}\right)$ is positive, a cylinder if $R\left(r_{0}\right)=0$, and convergent if $R\left(r_{0}\right)$ is negative. An elementary solution $R_{e}(r)$ could be obtained from (4.17) and the boundary conditions (4.19), (4.20) and an arbitrary given non-zero value for $R^{\prime \prime}(0)$. Then, the solution of $(4.17)-(4.20)$ is

$$
R(r)=c R_{e}(r)
$$




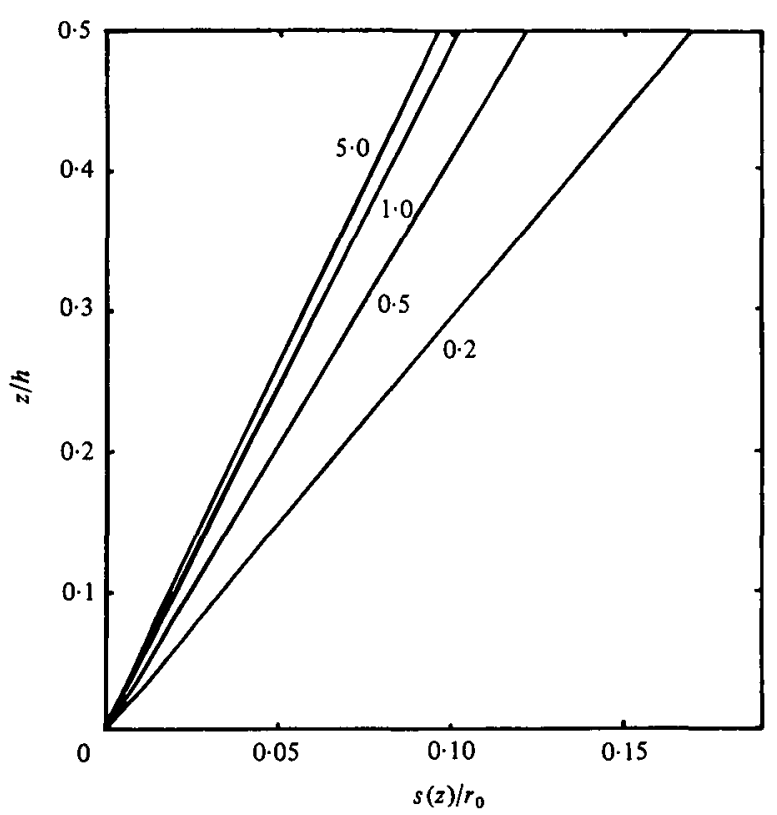

Figure 4. The boundary configurations of an isolated magnetic flux tube for different values of $A$, shown against the curves.

where the constant $c$ is determined from (4.18) as

$$
c=\frac{A q_{n}}{2}\left[8 \pi p_{0}\left(A^{2}+1\right)\right]^{\frac{1}{2}} /\left[\frac{3 A^{2}+1}{A^{2}+1} R_{e}\left(r_{0}\right)+r_{0} A^{2} R_{e}^{\prime}\left(r_{0}\right)\right] .
$$

Therefore, the condition of positive $R\left(r_{0}\right)$ requires

$$
\frac{3 A^{2}+1}{A^{2}+1}+r_{0} A^{2} \frac{R_{e}^{\prime}\left(r_{0}\right)}{R_{e}\left(r_{0}\right)}>0 .
$$

The results of elementary solution show that both $R_{e}\left(r_{0}\right)$ and $R_{e}^{\prime}\left(r_{0}\right)$ have the same sign, and condition (5.3) is always satisfied for the cases discussed in the present paper. The boundary configurations of flux tubes are shown in figure 4 for several typical cases. It can be seen that the flux tube is always divergent, and becomes increasingly so for larger values of $A$. The influence of atmospheric scale height on the boundary configuration can be seen from condition (4.13); the boundary function $s(z)$ is proportional to $\sin \left(\lambda_{n} z / h\right)$ for a finite value of $z / h$, for example $z / h \leqslant 0.5$ as in figure 4 . This means that the greater the scale height, the less divergent is the flux tube, and the configuration of the flux tube reduces to a cylinder in the limit of infinite scale height. Thus it is similar to the one-dimensional model of a magnetic flux tube confined in the uniform pressure atmosphere.

The approximation of large scale height permits us to assume that the boundary surface deviates only a little from a cylinder. This is the weak nonlinear case, and the free boundary problem reduces to a problem with fixed boundary. The set of equations (4.17)-(4.20) are solved by replacing the function $\exp (-z / h)-1$ in the asymptotic expression (4.24). This replacement is acceptable. Mathematically, the asymptotic expression has a smaller deviation of higher order, and it is shown that the difference between the solutions obtained 
by using the asymptotic expression and the exact function is also small for an elliptic partial differential equation. In fact, the form of the asymptotic expression (4.24) is the same as the component of the general expression (4.14), and the general problem could be similarly solved given a suitable condition at $z=z_{1}$. From the physical point of view, using the asymptotic expression means that we discuss a stratified atmospheric model with a temperature distribution which is nearly uniform, although not exactly constant.

In the present paper, we discuss the weak nonlinear problem with a free boundary. The results show the two-dimensional features of a magnetic flux tube, which may have many applications. A similar approach has been used to analyse other confined magnetic regions, such as the magnetic arch or plasma arch $(\mathrm{Hu} \& \mathrm{Li} 1986 a-c)$. The model of a magnetic field confined in the atmosphere with limited scale height will be discussed elsewhere. Understanding these nonlinear problems with a free boundary is important because the idea of an isolated magnetic flux region has extensive applications and is the basis of many further studies, for example, of the flow field, wave propagation, heat transfer and other processes in the flux tube.

This research was supported by the contract Mathematics-Physics 85060 of National Science Foundation of China. The author thanks the referee, Dr Philippa Browning, for her very helpful comments on this paper.

\section{REFERENCES}

Deinzer, W. G. 1965 Astrophys. J. 141, 548.

Deinzer, W. G., Hensler, G., Schussler, M. \& WeisshaAr, E. 1984 Astron. Astrophys. $139,426$.

Hv, W. R. 1981 Scientia Sinica, 24, 1680.

Hu, W. R. \& LI, J. G. $1986 a$ Acta Astron. Sinica, 27, 303. (In Chinese.)

Ho, W. R. \& LI, J. G. $1986 b$ Acta Astrophys. Sinica, 6, 254. (In Chinese.)

Hu, W. R. \& LI, J. G. 1986c Monthly J. Science, 31, 1780. (In Chinese.)

LÜST, R. \& SchlÜter, A. 1954 Zs. Astrophys. 34, 303.

Osherovich, V. A. 1984 Solar Phys. 94, 205.

PARKer, E. N. 1974 Astrophys. J. 191, 245.

Parker, E. N. 1979 Cosmical Magnetic Fields. Oxford University Press.

Spruit, H. C. 1981 The Sun as a Star (ed. S. Jordan), p. 385. NASA SP-450.

Wilson, P. R. 1977 Astrophys. J. 214, 611.

Yun, H.S. 1970 Astrophys. J. 162, 975. 